\title{
La psychosociologie : un cadre interprétatif de la fonction de tuteur dans un dispositif d'apprentissage par problèmes
}

\author{
Psycho-sociology as an interpretative framework of tutor role \\ in a problem-based learning course
}

Viviane VIERSET ${ }^{1}$, Denis BÉDARD ${ }^{2}$ et Jean Michel FoIDART ${ }^{3}$

1 Psychosociologue, Conseillère pédagogique du département de gynécologie-obstétrique, Centre hospitalier et universitaire et faculté de médecine, Université de Liège, Belgique

2 Centre d'études et de recherche en enseignement supérieur (CERES), Faculté d'éducation, Université de Sherbrooke (Québec), Canada

3 Département de Gynécologie-Obstétrique, Centre hospitalier et universitaire et faculté de médecine, Université de Liège, Belgique

Manuscrit reçu le 4 février 2009 ; commentaires éditoriaux formulés aux auteurs le 5 mai et le 26 octobre 2009 ; accepté pour publication le 23 novembre 2009

Mots clés :
Styles d'animation;
apprentissage
par problème;
petits groupes
d'apprentissage,
rôle de tuteur;
dynamique de groupe;
production;
facilitation;
régulation

Keywords:

Tutoring style; problem-based learning; small group learning; tutor's role;

\begin{abstract}
Résumé - Problématique : L'apprentissage par problèmes (APP) est une méthode de pédagogie active possédant son identité propre définie par des paramètres invariants. Toutefois, des variantes appropriées, argumentées et justifiées par les aspects intégratifs du contexte et de ses membres actifs (étudiants et/ou professeurs) peuvent être opportunes, pour autant que la méthode soit respectée dans ses fondements pédagogiques. La façon d'assurer la fonction de tuteur lors des rencontres avec les étudiants fait notamment particulièrement débat. Finalité : En s'appuyant sur une clarification des principes directeurs des approches de pédagogie active, la finalité de cet article est de proposer une typologie des styles d'animation en APP, afin de faciliter de « bonnes pratiques » de tutorat de la part des enseignants concernés, grâce à un ajustement de leur démarche d'animation en fonction des choix personnels et contextuels. Résultats : Des liens sont élaborés entre des concepts pédagogiques et des concepts psychosociologiques relatifs à la dynamique de groupes. Un outil permettant l'identification des styles d'animation en APP - et par extension applicable à tous les travaux en petits groupes s'inscrivant dans les principes de la pédagogie active - est proposé. Une telle typologie facilite la distinction des fondements essentiels («invariants »), des variations contextuelles («variants ») d'un groupe d'APP.
\end{abstract}

Abstract - Problematic: Problem Based Learning (PBL) is an active educational method that has its own specific identity defined by invariant parameters. However, appropriate variants, argued and justified by integrative aspects of the context and by its active members (students and/or professors) could be suitable as long as the method's educational basis is respected. Means to assure tutor's role during PBL sessions with students are a matter of 
group dynamics; regulation debate. Purpose: Based upon key conceptual frameworks of active-base education, the purpose of this article is to propose a classification of PBL tutoring styles, in order to promote "best practices" of tutoring from concerned professors, who adjust their tutoring method regarding personal and contextual choices. Results: Links are elaborated between educational and psycho-sociological concepts regarding groups dynamic. A tool allowing PBL tutoring style identification, and more generally in active-base education, is proposed.

\section{Introduction}

Généralement, dans nos institutions universitaires, l'implantation de méthodes pédagogiques actives se caractérise par la diminution de la place accordée aux cours ex cathedra, au profit d'activités d'enseignement et d'apprentissage en groupes restreints. Le présent article fait référence à l'une des méthodes actives les mieux connues en enseignement supérieur, l'apprentissage par problèmes (APP). L'APP, qui enchâsse les activités d'apprentissage au sein du processus de résolution efficace d'un problème, propose à de petits groupes d'étudiants de prendre connaissance d'une situation-problème, d'en comprendre les particularités et, ultimement, de tenter de l'expliquer et/ou de la résoudre avant toute intervention théorique du professeur ${ }^{[1]}$.

Ainsi, cette méthode s'appuie-t-elle sur la sollicitation des connaissances antérieures acquises par chaque étudiant du groupe, en les utilisant pour que le groupe construise ensemble de nouvelles connaissances adaptées à son niveau d'étude, sous l'œil vigilant d'un tuteur. Charlin ${ }^{[2]}$ et al. rappellent les principes opérationnels et les différents formats d'intervention des dispositifs se réclamant de l'APP. Dans la plupart des cas, le maintien des principes fonctionnels initiaux de l'APP dans l'enseignement supérieur signifie que ce travail d'apprentissage - individuel mais avec l'aide du groupe -, est construit par les étudiants au sein d'un groupe géré par un enseignant-tuteur et animé par un étudiantanimateur. Dans ce cadre, quatre rôles peuvent être dévolus aux étudiants pour chacune des séances de travail en groupes (tutorial) : le secrétaire inscrit sur un tableau les éléments pris en compte dans l'analyse du problème, afin que chaque participant puisse suivre librement le déroulement sans prendre de notes; le scribe transcrit sur papier les éléments significatifs du tableau, dans un document distribué à chaque membre du groupe en fin de séance ; le rapporteur assure les communications entre le groupe et les responsables du programme et veille à la disponibilité des ressources didactiques; enfin, l'animateur gère les étapes de la démarche de résolution du problème, en suscitant la participation, en régularisant les interactions et favorisant l'expression des consensus (ou des dissensus).

Si cette dernière fonction d'animation n'est pas assumée, le tuteur doit la prendre en charge. Il devient alors tuteur-animateur, ce qui arrive couramment dans les groupes qui maitrisent insuffisamment la méthode d'APP. En effet, l'étudiant, même s'il ne récuse pas la fonction, éprouve souvent de grosses difficultés à l'assurer tout en s'appropriant simultanément une matière nouvelle et complexe. Nous avons ainsi observé à maintes reprises la dilution de cette attribution lorsque l'étudiant est luimême en apprentissage et en recherche de solution au problème posé. Il est donc conseillé que soient fournis, à la fois aux étudiants-animateurs et aux tuteurs-animateurs, appelés à reprendre éventuellement la fonction initialement dévolue à l'étudiant, les moyens d'appréhender efficacement ce rôle.

À titre d'exemple, dans le cadre de la formation d'ingénieurs à l'université catholique de Louvain (Belgique), le tuteur encadre plusieurs groupes de travail simultanément. De Theux ${ }^{[3]}$ et al. expliquent qu'il est alors essentiel que les étudiants responsables de l'animation dans les différents groupes «jouent le jeu » de manière la plus efficace possible. Pour cela, au préalable, les étudiants sont formés à leur fonction d'animation par des exercices structurés de simulation, dans le cadre d'une formation 
nommée APP 0 (APP zéro), organisée durant la première semaine de cours de leur cursus académique. Ainsi, insistons-nous sur cette fonction d'animation que doit en principe assurer l'étudiant concerné ou que devra prendre le tuteur - devenu alors tuteuranimateur -, le cas échéant. Car, si le tuteur demande à un étudiant d'assurer cette fonction, il doit pouvoir le guider dans cette tâche, voire l'assurer lui-même. L'étude descriptive que nous proposons dans cet article s'attache spécifiquement à préciser cette fonction de tuteur conscient de sa responsabilité d'animation.

L'implantation des méthodes actives en pédagogie universitaire a en effet pour conséquence majeure d'exiger l'appropriation de nouvelles compétences de la part de l'enseignant. Il ne lui est plus seulement demandé d'être « un bon pédagogue » au sens communément admis, c'est-à-dire « de bien expliquer son cours » ou «de s'exprimer de manière attrayante ». Il lui est aussi demandé d'être capable de guider un groupe d'étudiants, qui échafaudent de nouveaux apprentissages grâce à l'activation de leurs connaissances antérieures, vers la réalisation efficace d'un travail commun. Ainsi, parallèlement à la composante pédagogique de l'enseignant universitaire, s'ajoute la composante psychosociologique centrée à la fois sur la gestion (en tant que tuteur) et sur l'animation de groupes restreints (en tant qu'animateur). Dès lors, nous insistons sur ces deux composantes.

Une communication d'information révèle une composante pédagogique quand elle réorganise adroitement les connaissances ou la manière de les aborder, afin d'en faciliter l'appropriation. Par ailleurs, la composante psychosociologique travaille sur l'analyse des moteurs et des freins d'un fonctionnement groupal. Autrement dit, le groupe social d'APP, formé des apprenants et du tuteur, est observé en tenant compte des rôles effectivement pris par ses individus constitutifs. L'étude de la dynamique des groupes restreints et celle de l'animation de groupe sont des sujets de la psychosociologie, tels qu'ils sont notamment abordés en management ou en psychologie des entreprises.
Nous intégrons ces deux composantes dans une même typologie, forcément réductrice. Car, comme Morin ${ }^{[4]}$ le souligne, «la pensée complexe est une pensée qui cherche à la fois à distinguer - mais sans disjoindre - et à relier. », ce à quoi il ajoute : «L'univers n'est pas soumis à la souveraineté absolue de l'ordre, il est le jeu et l'enjeu d'une dialogique entre l'ordre, le désordre et l'organisation. » Le modèle, la typologie et le schéma sont des outils dont on se sert pour mieux comprendre la réalité mais ils ne sont pas la réalité ; la réalité se crée en permanence et de manière différente, toujours en relation d'équilibre/déséquilibre avec le contexte de vie.

Dans le cadre de la présente contribution, nous proposons successivement : 1) de rappeler les paramètres identitaires - ou principes directeurs - de la pédagogie active; 2) de préciser la notion de styles d'enseignement (actif, permissif, incitatif et transmissif) et de mettre en perspective cette typologie avec plusieurs points qui font actuellement débat concernant les rôles du tuteur dans le cadre de dispositifs d'APP; 3) enfin, en nous appuyant sur une perspective psychosociologique appliquée à la dynamique des groupes restreints, telle qu'elle a été développée dans les années 70, et notamment sur le concept de «tripartition des rôles dans un groupe de travail », de proposer une clarification des rôles du tuteur (production, facilitation et régulation), en les mettant en correspondance avec les styles d'animation.

\section{Les paramètres identitaires - ou principes directeurs - de la pédagogie active}

\author{
Les orientations de la "nouvelle pédagogie » \\ à partir des travaux John Dewey
}

Les racines de la pédagogie active se sont creusées dans le sol philosophique de la «nouvelle pédagogie ». Le travail de John Dewey ${ }^{[5]}$, philosophe, psychologue et pédagogue américain du début du $\mathrm{XX}^{\mathrm{e}}$ siècle, a favorisé l'introduction de ce concept qui va bouleverser l'approche pédagogique utilisée 
jusqu'alors. Ses propositions soulignent l'importance de l'apprentissage par la pratique - «learning by doing ». Pour Dewey ${ }^{[5]}$, le sujet doit percevoir que l'activité d'apprentissage qui lui est proposée est utile pour lui. Barr et Tagg ${ }^{[6]}$ soulignent qu'il s'agit pour les institutions de passer du paradigme d'enseignement (teaching paradigm) au paradigme d'apprentissage (learning paradigm). Les méthodes actives ont pour but non seulement de donner la possibilité à l'étudiant d'agir et de participer, mais aussi de lui fournir des occasions de mobiliser, de modéliser, d'actualiser et d'évaluer l'ensemble de ses capacités dans l'action proposée par l'enseignant.

La naissance de cette nouvelle structuration pédagogique s'organise autour d'un changement d'attitude entre les partenaires de travail, c'està-dire principalement entre les enseignants et les étudiants. Anzieu et Martin ${ }^{[7]}$ ont défini le concept d'attitude comme rendant compte de nos «prédispositions à agir d'une certaine manière vis-à-vis d'autrui »; il s'agit aussi d'un «matériel psychologique, conscient et inconscient, qui détermine nos comportements vis-à-vis d'autrui ». Pour introduire ce changement d'attitude, il est incontournable que des dispositifs organisationnels et institutionnels, instituant les fonctions d'étudiants-animateurs et d'enseignants-tuteurs, soient mis en place. Leur finalité est de faciliter l'implantation du processus «actif» en permettant l'opérationnalisation des paramètres identitaires des pédagogies actives, tels que les rappelle Vierset ${ }^{[8]}$ :

- mise en place par l'institution de conditions favorables à des processus d'apprentissage qui déterminent l'étudiant comme acteur, sinon comme compositeur, responsable individuellement de sa formation. Une logique inductive et des apprentissages divergents créent un espace stratégique ouvert, laissant la place aux potentiels de gestion, d'innovation et de créativité intellectuelle de l'ensemble des individus du groupe;

- mise en place par l'institution d'un système d'acquisition de compétences, justifiant la formulation d'objectifs de formation cohérents par l'enseignant. Les objectifs d'apprentissage révèlent les question- nements et les besoins rencontrés par les apprenants pour résoudre le problème (au bout du compte, la majeure partie des objectifs d'apprentissage devraient correspondre aux objectifs de formation); - explicitation des liens à construire par l'étudiant entre ses connaissances antérieures et l'appropriation des nouvelles connaissances. En adoptant une vision intégrée et interdisciplinaire des connaissances, ce type de travail constitue un exercice à la discrimination et à la généralisation des données mises en relations ;

- mobilisation des ressources internes (cognitives, affectives et procédurales) et externes (documentaires et humaines) des étudiants, lors d'exercices de simulation ou de résolution de problèmes réels, signifiants et contextualisés, qui développent le transfert des apprentissages (contextualisation / décontextualisation / recontextualisation);

- développement d'une dimension sociale et collaborative en groupes restreints, favorisant les processus de concertation et d'expression-confrontation entre les apprenants (conflit socio-cognitif), en vue de négocier une solution commune. Celle-ci s'échafaude lors d'un travail groupal et d'un travail individuel préliminaire ;

- ouverture à la diversité des approches - qualitatives et quantitatives - fondées sur des documents scientifiques, avec des références précises et de nombreuses ressources documentaires ciblées mises à disposition par l'enseignant ;

- ouverture à l'anticipation, voire à la prise de risque intellectuel, stratégique ou social dans ce processus non linéaire, organique et parfois insécurisant, qui nécessite un encadrement par l'enseignant, potentiellement souple, positif et valorisant pour les apprenants ;

- développement par l'enseignant d'un encadrement structurant pour les apprenants, favorisé par des recadrages et des synthèses systématiques. $\mathrm{Au}$ terme du parcours, le tuteur - voire un étudiant -, propose une synthèse des notions acquises par le groupe ;

- organisation par l'enseignant de rétroactions 
argumentées, d'évaluations du parcours et de validations de l' « œuvre » réalisée, c'est-à-dire des propositions de solution au problème posé.

La contribution de la perspective socio-constructiviste

D'une manière générale, le courant de la pédagogie active s'est fortement nourri de la perspective socioconstructiviste de l'apprentissage, qui met l'accent sur le rôle des interactions sociales multiples dans la construction des savoirs. Cette perspective est ellemême issue de la convergence de plusieurs courants théoriques. Ainsi Albert Bandura, psychologue canadien, est l'auteur d'une théorie sociale cognitive de l'apprentissage ${ }^{[9]}$, qui met l'accent sur l'importance du concept d'auto-efficacité (self-efficacy) chez l'apprenant. Selon lui, l'opportunité de pouvoir observer un individu similaire à soi-même exécuter avec succès une activité donnée constitue une source d'information importante influençant la perception d'auto-efficacité. En précurseur du constructivisme, Piaget ${ }^{[10]}$ propose un modèle contemporain du développement de l'apprentissage et précise que l'on ne connaît un objet qu'en agissant sur lui et en le transformant. Il met l'accent sur l'importance déterminante de l'interaction sujet-environnement dans l'apprentissage. Vygotsky ${ }^{[11]}$ élargit cette idée en privilégiant la dimension sociale des connaissances qui se construisent dans l'action et la réflexion, « les cognitions émergeant dans et par l'interaction sociale ». Dans la perspective socio-constructiviste, il y a conflit socio-cognitif lorsque, dans une même situation, différentes approches cognitives d'un même problème sont construites socialement. Une telle situation génère une dynamique interactive, caractérisée par une coopération active, avec prise en compte de la réponse ou du point de vue d'autrui, et recherche, dans la confrontation cognitive, d'un dépassement des différences et des contradictions pour parvenir à une réponse commune.

Ainsi, la perspective socio-constructiviste, défendue par de nombreux philosophes et psychologues, confère une forte légitimité à cette toute ré- cente promotion de l'étudiant actif et elle fournit des repères importants pour guider le changement de paradigme pédagogique actuel dans les universités. Plusieurs applications pédagogiques peuvent ainsi être déduites de ces différents éclairages :

- au plan général, un cadre de travail «actif» a avantage à être développé graduellement, à partir d'une perspective sollicitant une activité et une autonomie relativement limitées au départ, vers une perspective de plus en plus large, faute de quoi peuvent être redoutées une démission et une perte d'efficacité de l'étudiant devant l'étendue de la tâche exigée par l'enseignant ;

- d'une façon plus spécifique, l'un des intérêts majeurs d'activités conduites en groupes restreints, telles qu'elles sont par exemple organisées au cours de l'APP, est de permettre l'exploitation pédagogique des conflits socio-cognitifs résultant des interactions sociales multiples qui se développent à l'intérieur du groupe étudiants/tuteur. De telles conditions permettent en effet une expressionconfrontation des résultats de recherche ou des solutions proposées et argumentées dans/et par le groupe d'étudiants, à la suite de leur recherche individuelle.

Imaginons par exemple qu'au début d'une séance, deux étudiants proposent des solutions différentes; un troisième étudiant est d'accord avec une partie mais pas la totalité d'une des démonstrations ; un quatrième va proposer un schéma... Plutôt que dire « ça, c’est la bonne réponse », le tuteur préférera dire : «quels sont les points communs entre les quatre propositions? Sur quoi sont-elles fondées? Où se trouve la faille dans tel raisonnement? Qu'en pensent les autres ?... ». Il contribuera ainsi à opposer les échanges dans le groupe, favorisera la controverse et le débat pour l'enrichir des propositions de chacun. Il est essentiel de rappeler que ce conflit, appréhendé simultanément au niveau social et au niveau cognitif, constitue un des paramètres invariants à privilégier lors d'une animation de style actif, telle que nous la définissons plus loin.

Au total, les chercheurs à l'origine des différents cadres conceptuels évoqués précédemment ont graduellement ouvert la voie aux modèles 
d'enseignement et d'apprentissage qui sont à la base des pédagogies actives. Les pédagogies actives cherchent à donner l'occasion aux apprenants d'élaborer une construction personnelle des connaissances en suivant leur propre schéma d'appropriation et non uniquement le schéma d'appropriation de l'enseignant. Bachelard ${ }^{[12]}$ rappelle que « tout enseignement reçu est un empirisme alors que tout enseignement donné est un rationalisme ». Bref, cette orientation pédagogique apporte un souffle neuf invitant à remettre en cause les méthodes académiques expositives, dans le cadre desquelles l'étudiant écoute le discours de l'expert enseignant. En effet, même lorsque les savoirs sont présentés à l'étudiant de manière expositive, l'étudiant doit construire ses connaissances en faisant référence à ses propres schémas et à ses connaissances antérieures $^{[13]}$. Dans le cas d'un cours magistral, la logique et la rationalité propres de l'enseignant créent un contexte directif et contraignant qui peut être plus ou moins favorable au processus d'apprentissage. Dans le cadre de dispositifs de type APP, on fait l'hypothèse que la construction personnelle de l'étudiant est moins sujette aux a priori de l'enseignant et à la rationalité, par nature personnelle, de ce dernier.

\section{Les styles d'enseignement et les styles d'animation dans le cadre d'un dispo- sitif d'apprentissage par problèmes.}

De nombreux auteurs se sont intéressés aux styles d'animation des tuteurs dans le cadre de dispositifs d'APP. Leung et al. ont développé et validé un instrument qui évalue les styles d'animation de tutoriaux d'APP ${ }^{[14]}$. Notre choix personnel est d'orienter notre recherche vers la construction d'une typologie des styles d'animation en APP, en l'adossant à trois contributions complémentaires. Parmi ces dernières, deux sont de nature conceptuelle, issues respectivement, d'une part d'une catégorisation pédagogique élémentaire décrivant différents styles d'enseignement et, d'autre part, d'une approche psychosociologique appliquée à la dynamique des groupes restreints; la troisième est de nature empirique et s'appuie sur l'expérience acquise depuis une dizaine d'années par l'une d'entre nous (VV) dans le cadre de plusieurs groupes de travail dédiés à l'APP à la faculté de médecine de l'université de Liège.

Une catégorisation des styles d'enseignement

Une catégorisation, des styles d'enseignement, ellemême inspirée des recherches conduites par Blake et Mouton sur la notion de management dans les années $60^{[15]}$, a été proposée en 1984 par Therer et Willemart ${ }^{[16]}$. Chaque style d'enseignement peut se définir à partir des différents types d'interactions qui peuvent être observées entre l'enseignant et les étudiants qui constituent une classe.

\section{Le style actif}

Le style actif caractérise les interventions de l'enseignant qui régule et facilite le débat entre les étudiants de la classe. Il valide/invalide leur démarche d'apprentissage et leur solution finale. Les interactions prenant la forme d'expression/confrontation sont majoritaires entre les étudiants. Le tuteur est alors «gestionnaire» des relations dans le groupe, tout en veillant à la construction d'un chemin menant vers une solution efficace. Les invariants de la pédagogie active sont dans ce cas identifiés clairement.

\section{Le style transmissif}

Le style transmissif caractérise les interventions d'un enseignant qui progresse seul. L'essentiel du style transmissif porte sur le déroulement expositif de la stratégie cognitive de l'enseignant face aux étudiants. Dans certains cas, un enseignement transmissif est fondé sur la résolution d'un problème. On parle alors d'enseignement par problème (EPP). On constate ici une faible interactivité, voire l'absence de toute interaction, entre les étudiants. Si elles existent, de sporadiques communications sont 
entretenues avec l'enseignant. Dans le cas où les interactions entre les étudiants ne sont pas souhaitées lors de l'exposé, l'enseignant devient exclusivement un « orateur ».

\section{Le style incitatif}

Le style incitatif caractérise les interventions de l'enseignant qui tente de faire s'exprimer les étudiants. De nombreux questionnements individuels et des réponses à des questions cognitives personnelles des étudiants forment la trame communicationnelle du groupe. Le style incitatif insiste sur la construction des questions (questions ouvertes/fermées) et sur la réponse aux attentes des étudiants pris individuellement dans le groupe. Le professeur a le souci de faire participer les étudiants dans les groupes constitutifs en sollicitant des réponses et des avis. Les interactions se font alors majoritairement entre l'enseignant et l'un ou l'autre des étudiants. Les autres membres de la classe demeurent plus ou moins passifs selon les situations. Privilégiant son rôle d'expert, l'enseignant est surtout une «personne ressource » à qui les étudiants peuvent poser des questions. Ce style hybride entre le style actif et le style transmissif donne l'occasion de développer des comportements interactifs plus ou moins performants selon la forme et le fond des questions proposées.

\section{Le style permissif}

Le style permissif caractérise les interventions d'un enseignant qui « laisse faire» les étudiants sans encadrement. On n'observe aucune interaction des étudiants vers l'enseignant mais uniquement entre les étudiants. Dans ce cas, l'enseignant reste en dehors de la classe sans intervenir. Il devient alors un « observateur passif ».

Ces différents styles peuvent être schématisés sur un diagramme qui prend en compte, sur deux axes, l'intérêt que l'enseignant accorde respectivement à l'apprenant ou à la matière (figure 1).

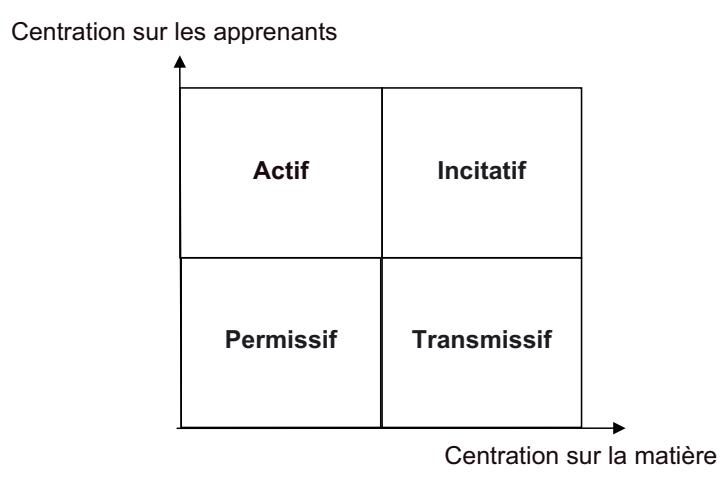

Style transmissif : centré davantage sur la matière.

Style incitatif : centré à la fois sur la matière et sur les apprenants.

Style actif : centré davantage sur les apprenants.

Style permissif : très peu centré, tant sur les apprenants que sur la matière.

Fig. 1. Les styles d'enseignement selon Therer et Willemart ${ }^{[14]}$

Un débat sur les rôles du tuteur dans un dispositif d'apprentissage par problèmes

De nombreux résultats et analyses soulignent que la qualité du tutorat est un élément fondamental $\mathrm{du}$ bon fonctionnement des dispositifs d'APP et qu'il est un élément critique de la construction des apprentissages $^{[17-21]}$, ce qui invite à préciser les caractéristiques de cette fonction.

\section{L'influence du style de tutorat sur les apprentissages des étudiants dans un dispositif d'APP}

Plusieurs travaux ont cherché à cerner les effets respectifs de l'expertise de contenu ou de l'expertise de processus sur les interactions entre les étudiants dans un tutorial d'APP car les tuteurs sont généralement des experts du contenu souvent sollicités par l'institution pour prendre en charge un tutorat en APP. Caplow ${ }^{[22]}$ et al. soulignent que les tuteurs devraient avoir des connaissances en animation de groupe suffisantes afin de guider le groupe d'apprenants vers les objectifs de formation et d'apprentissage visés; comme nous l'avons souligné précédemment, il arrive fréquemment que l'étudiant-animateur élude tout ou partie de ce rôle. Silver et Wilkerson ${ }^{[22]}$ ont identifié plusieurs types 
d'intervention qui constituent des facteurs négatifs et stressants : c'est le cas lorsque le tuteur dirige une question vers un étudiant en particulier en sollicitant explicitement une réponse de sa part et non pas de l'ensemble du groupe ou encore lorsqu'il formule trop de questions consécutives. Ils ont également établi que, lorsque les tuteurs sont des experts du contenu disciplinaire, ils parlent plus souvent, plus longtemps, formulent davantage des questions fermées suggérant des réponses brèves et peu approfondies et, enfin, répondent «spontanément » aux questions individuelles des étudiants en focalisant les discussions sur leurs propres interventions. De telles interventions sont clairement davantage en accord avec une approche pédagogique centrée sur l'enseignement qu'avec une approche centrée sur l'apprentissage ${ }^{[24]}$ et favorisent l'adoption par les étudiants de démarches de résolution du problème conformes à celles du tuteur. Elles témoignent d'un style directif ou d'un style incitatif, selon la typologie décrite précédemment et s'opposent à l'attitude passive, non interventionnelle et non directive - laissant une totale liberté aux étudiants - qui correspond au style permissif.

À l'inverse, plusieurs travaux ont établi que les tuteurs qui privilégient un questionnement ouvert, en lien avec un style actif, en exigeant un raisonnement approfondi, plutôt que le recours à des questions plus ou moins fermées (style incitatif) ou à des « capsules » magistrales (style transmissif), favorisent davantage le développement d'habiletés à la résolution de problèmes et la réflexion ${ }^{[20,25-27]}$.

Certains auteurs, tels que Bowman et Hughes ${ }^{[18]}$ recommandent que les tuteurs délaissent leur rôle d'expert de contenu au profit de celui d'animateur, de manière à favoriser les processus d'interaction socio-cognitive, en facilitant l'expression et l'argumentation entre les étudiants. Néanmoins, Lebrun ${ }^{[28]}$ observe qu'en pratique, quand les tuteurs donnent certaines informations (« mini-cours ») pour débloquer une situation figée, et bien que de ce fait ils « court-circuitent» le processus complet d'apprentissage autonome, ils communiquent conjointement une mise au point et/ou une rétro-action favorables au transfert, ce qui contribue à l'activité de l'étudiant et donc à son apprentissage. De telles interventions, à conditions d'être brèves, non systématiques et effectuées avec opportunité, peuvent assurer un effet catalyseur bénéfique à une dynamique de travail au sein d'un groupe. Ainsi, le «bon tuteur» pourrait être un expert de contenu doublé d'un expert en démarche d'APP, entretenant un rapport d'équilibre entre ces deux rôles qu'il maîtriserait l'un et l'autre.

\section{Les caractéristiques $d^{\prime}$ un " bon tuteur " de style actif dans un dispositif d'APP}

Les paramètres identitaires - ou principes directeurs - de la pédagogie active, évoqués précédemment, permettent de cerner les caractéristiques d'un tuteur de style actif et efficace. Ainsi, Steinert ${ }^{[20]}$ souligne qu'un «bon tuteur» encourage les échanges entre étudiants dans un environnement et un climat favorables aux apprentissages. En cohérence avec la notion de l'influence favorable des conflits socio-cognitifs sur l'apprentissage, il valorise l'expression et la confrontation des données en valorisant les propositions divergentes. D'autres auteurs mentionnent l'importance d'établir un bon contact avec les étudiants tout en favorisant la coopération et les interactions entre les membres du groupe. En d'autres termes, à l'inverse des méthodes expositives, plus académiques, le tuteur intervient surtout sur la gestion du groupe et sur la progression du travail (synthèses de ce qui a été dit, reformulations, demandes de précisions, questionnement ouvert sur le processus de raisonnement suivi, etc.), mais beaucoup moins sur le contenu d'expertise qu'il appartient aux étudiants de construire euxmêmes, grâce aux ressources documentaires mises à leur disposition.

Le «bon tuteur» veille par exemple à ce que les apprenants ne s'écartent pas du sujet en cherchant à trop approfondir une notion singulière et non significative; pour ce faire, il interviendra en disant : «Pensez-vous que ce soit utile à la résolution de votre problème ? » plutôt que : «Cela n'a pas d'importance, continuons... ». Il préfère ne pas répondre 
à une question de contenu en retournant la question à l'interlocuteur, voire au groupe, en disant : «Qu'en pensez-vous ?». Il s'agit d'encourager la réflexion pour susciter des hypothèses explicatives ou résolutives, selon les cas, qui seront à vérifier plus tard. Ce n'est qu' au terme de la séance de retour que le tuteur avalise les propositions des étudiants ou les dirige vers un expert du contenu, en consolidant ou en invalidant les solutions proposées. Une telle démarche semi-directive est en accord avec les principes directeurs d'un enseignement de style actif.

Elle est également en forte cohérence avec la perspective de l'apprentissage et de l'enseignement contextualisés authentiques (AECA), telle qu'elle est proposée par Bédard, Frenay et leurs collègues ${ }^{[29,30]}$ et qui s'appuie sur deux principes : la contextualisation des apprentissages et le compagnonnage cognitif ${ }^{[29,30]}$. La perspective de l'AECA est l'un des éléments clés du cadre conceptuel qui inspire les activités d'enseignement et d'apprentissage au cours des tutoriaux en APP ${ }^{[31]}$. D'un point de vue empirique, cette perspective a pris naissance autour des réponses à apporter aux questions que soulève le transfert des connaissances chez l'étudiant. D'un point de vue théorique, elle prend assise dans les préceptes socio-cognitivistes et socioconstructivistes.

Le principe de la contextualisation concerne l'adéquation entre la situation d'apprentissage et les contextes socio-professionnels. Lorsque cette exigence est satisfaite, par exemple dans le cadre d'un dispositif de type APP, les étudiants font plus facilement appel à des opérations cognitives complexes qui nécessitent un partage d'informations et une communication sociale, ce qui facilite la résolution efficace du problème et le transfert ultérieur des connaissances acquises ${ }^{[32]}$.

Le compagnonnage cognitif postule que la construction du savoir chez l'apprenant est facilitée et favorisée, voire médiatisée, par le travail d'accompagnement offert par l'enseignant. Cette orientation des rôles de l'enseignant fait notamment référence à la stratégie d'échafaudage (scaffolding) qui propose aux étudiants de réaliser une tâche complexe, le support qui leur est offert variant selon leur degré de maîtrise des compétences; le professeur peut assumer lui-même une partie de la tâche compte tenu de la compétence des étudiants. De façon large, ce concept renvoie au support offert par le tuteur à l'ensemble des étudiants d'un groupe, sans toutefois que ses interventions ne deviennent omniprésentes comme l'impliquerait un style incitatif.

Un «bon tuteur» peut également rendre explicite l'importance de la responsabilité individuelle de chaque apprenant ${ }^{[26,27,33]}$. L'engagement personnel de chaque étudiant, en lien avec l'exercice de sa prise de responsabilité individuelle au sein d'un travail de groupe, constitue un élément facilitant la progression du travail. L'adoption d'une attitude positive et valorisante constitue un facteur essentiel qui influence la motivation à apprendre et augmente la qualité des apprentissages réalisés ${ }^{[27]}$ par tous les individus du groupe.

Gilkison $^{[24]}$ souligne que, lorsque les questionnements du tuteur, dans le cadre d'un style d'intervention de type actif, permettent de formuler un problème authentique qui capte l'attention des étudiants envers l'objet d'étude, une argumentation de haut niveau taxonomique et une analyse en profondeur sont plus facilement développées par ces derniers. La complexité du problème suscite et favorise les interactions sociales dans le groupe et hors du groupe. Les objectifs reliés à des capacités transversales telles que la recherche, l'analyse, l'argumentation, la confrontation d'idées, la collaboration, la schématisation, la prise de risque, etc. sont ainsi plus facilement atteints. La communication sociale, la négociation, la prise de parole, l'interview de personnes ressources, etc. sont améliorées.

Dans une étude récente menée par Mete et Sari ${ }^{[21]}$ avec des étudiants en sciences infirmières, ceux-ci mentionnent l'importance des questions soulevées par le tuteur pour conduire les membres du groupe à réfléchir sur les relations à établir entre les connaissances antérieures et les connaissances nouvellement acquises, de même que sur les liens à organiser lors de leur application. Les étudiants ont également souligné l'importance des rétroactions et 
des synthèses engageant le processus de conceptualisation (nommé ici décontextualisation), ainsi que l'importance du processus de validation de la solution proposée soumise au tuteur. Ces deux invariants de la pédagogie active ont permis aux étudiants de cibler les contenus les plus importants et de réguler leur processus d'apprentissage. À l'issue d'une récente étude qualitative consacrée à l'animation du tuteur, Papinczak ${ }^{[31]}$ et al. ont comparé métaphoriquement le groupe d'étudiants à un orchestre symphonique; dans une telle perspective, tel un chef d'orchestre, le tuteur doit, par ses interventions, guider le groupe vers une harmonie socio-cognitive.

Enfin, un «bon tuteur» de style actif ne s'interdit pas d'adapter ses interactions avec les étudiants du groupe, en recourant judicieusement, de façon flexible, à des interventions qui relèvent plutôt des trois autres styles (transmissif, incitatif, permissif), lorsque des conditions non optimales y invitent (contexte peu facilitant, nombre élevé d'étudiants, désintérêt pour l'apprentissage manifesté par plusieurs, etc. ). La notion de style d'animation doit donc être comprise comme décrivant le style dominant employé par un enseignant donné. Par ailleurs, si l'animation d'un groupe par un tuteur est « tuteurdépendante », il importe de retenir qu'elle est également «groupe-dépendante » et aussi «situation contextuelle-dépendante ». Elle ne dépend pas exclusivement des caractéristiques personnelles d'animation des tuteurs ${ }^{[34]}$.

\section{Clarification du rôle de tuteur à partir de l'éclairage d'une approche psychosociologique appliquée à la dynamique des groupes restreints}

Comme cela a été rappelé précédemment, le cadre conceptuel de la «pédagogie active », selon lequel on examine aujourd'hui les rôles respectifs du tuteur et des étudiants ainsi que leurs interactions au sein d'un groupe d'APP, prend en compte de façon privilégiée les orientations suggérées par les perspectives cognitiviste et socio-constructiviste de l'apprentissage et par la perspective de l'enseignement et de l'apprentissage contextualisés authentiques. Sans remettre en cause d'une quelconque manière ces orientations majeures, la présente contribution souhaite au contraire exposer l'intérêt qu'il y a à continuer de prendre en compte les acquis de la perspective psychosociologique en éducation et notamment le concept de tripartition des rôles appliqué à la dynamique des groupes restreints. Développés depuis une trentaine d'années, ils peuvent selon nous être transposés de façon très pertinente à la problématique de l'animation au sein des groupes d'APP. Nous les avons nous-mêmes exploités pour tenter d'élaborer des solutions face aux problèmes liés à une hétérogénéité des pratiques de tutorat, telle qu'elle était observée dans notre institution et que les étudiants dénonçaient. Nous rapporterons brièvement cette expérience pour introduire quelques principes directeurs visant à clarifier le rôle de tuteur, que nous avons élaborés à partir de la perspective psychosociologique.

\section{Une hétérogénéité des pratiques de tutorat} observée dans un milieu institutionnel

Depuis une dizaine d'années, la faculté de médecine de l'université de Liège, dont le curriculum pré gradué fait une large part à l'APP, organise plusieurs groupes de travail dédiés à l'analyse et à l'amélioration du dispositif d'APP. Le fonctionnement de ce dispositif est maintenant rôdé et satisfait l'ensemble des acteurs. Toutefois, lors des évaluations et comme dans plusieurs autres milieux académiques où l'APP a été implanté, les étudiants expriment de manière récurrente un malaise, parfois ressenti comme une profonde injustice et générateur de stress lors de leur apprentissage. Ils font état d'une «trop » grande hétérogénéité des pratiques d'animation des différents tuteurs d'APP d'un groupe à l'autre, dans le cadre d'un même cours. Ils rapportent par exemple que «certains tuteurs donnent des cours, que d'autres donnent des «tuyaux » (des questions) d'examen et que d'autres se taisent tout le temps en hochant de la tête ». Par ailleurs, les tuteurs formulent également un besoin de clarification de leur fonction d'animation. 
En tant que conseillère pédagogique, l'une d'entre nous (VV) a été sollicitée pour une intervention, afin de tenter de résoudre ce problème devenu délicat. Dans un premier temps, nous avons élaboré une grille d'observation des styles d'animation en APP, qui correspond à une transposition de la typologie des styles d'enseignement de Therer et Willemart au cadre spécifique d'un groupe d'APP; elle est présentée en figure 2. Cette grille représente les différents styles d'animation possibles en APP selon deux axes. L'axe du développement des compétences professionnelles constitue la priorité des interventions dans le style actif tandis que l'axe du développement des contenus constitue la priorité dans le style transmissif. Le style incitatif tente de gérer les deux dimensions vaille que vaille tout en étant une forme hybride, hésitant entre ces deux dernières formes d'animation. Le style permissif laisse les interactions se faire d'elles-mêmes, sous le couvert de l'observation externe du tuteur. Cette typologie peut être assimilée à une clef d'identification des styles d'animation adoptés par un animateur dans le cadre d'un dispositif d'APP observé sous l'angle des interactions sociales au sein du groupe.

Puis, nous avons procédé, au cours de deux années académiques successives (2007-08 et 2008-09) à une observation des groupes d'APP, à l'aide de cet outil. Au sein des 35 groupes observés, mis à part quelques interventions ponctuelles de la part d'étudiants-animateurs ou secrétaires, le rôle d'animation, en principe dévolu à un étudiant, a été effectivement et régulièrement assuré par les tuteurs :

- trois tuteurs ont adopté simultanément le style transmissif et le style incitatif ;

- deux tuteurs ont majoritairement adopté le style permissif ;

- vingt-trois tuteurs ont majoritairement adopté le style incitatif ;

- sept tuteurs ont majoritairement adopté le style actif.

Ainsi, dans le cadre de cet inventaire descriptif des animations effectivement développées dans un dispositif fonctionnel d'APP, il a été constaté que lorsque l'animation n'est pas assurée par l'étudiant- animateur et que, par défaut, les tuteurs doivent prendre en charge ce rôle, ils recourent aux interactions sociales caractéristiques des quatre styles d'enseignement. La plupart recourent majoritairement à un seul des quatre styles. Seulement un cinquième des tuteurs utilise majoritairement le style actif. D'autres ont recourt à plusieurs styles lors d'une même séance. Nous avons observé que ce métissage, qui pourrait être adéquat, entretient cependant souvent une confusion entre les quatre styles; il est le fait à la fois de tuteurs débutants et de tuteurs plus expérimentés.

La notion de tripartition des rôles dans un groupe de travail

Dans les années 70, Palmade ${ }^{[35]}$, psychosociologue expert en psychologie des organisations, distingue, au terme d'une recherche concernant l'efficacité des groupes de travail, trois rôles qu'un animateur pourrait assumer lors d'une réunion. Ceux-ci sont explicités dans le tableau I et font référence à une tripartition des rôles d'animation selon une perspective psychosociologique.

Cette distinction de rôles étant explicitée, il ne s'agit pas seulement de demander aux tuteurs un exercice de classement de leurs interventions personnelles lors d'une animation de groupe. Il s'agit de leur proposer de prendre conscience de cette tripartition afin d'adopter les rôles pertinents, favorisant les confrontations cognitives argumentées des étudiants du groupe et leur cheminement vers l'atteinte de leurs objectifs d'apprentissage. Comme le précise en effet Saint-Arnaud ${ }^{[35]}$, l'animation est l'art de susciter une conscience de groupe chez chacun des membres, dans le but de favoriser la poursuite d'un objectif commun.

Une proposition d'outil d'auto-évaluation et de supervision pour la fonction de tuteur de style actif dans un dispositif $d$ 'apprentissage par problèmes

La contribution spécifique que nous proposons au terme de cet article vise à clarifier les interventions 


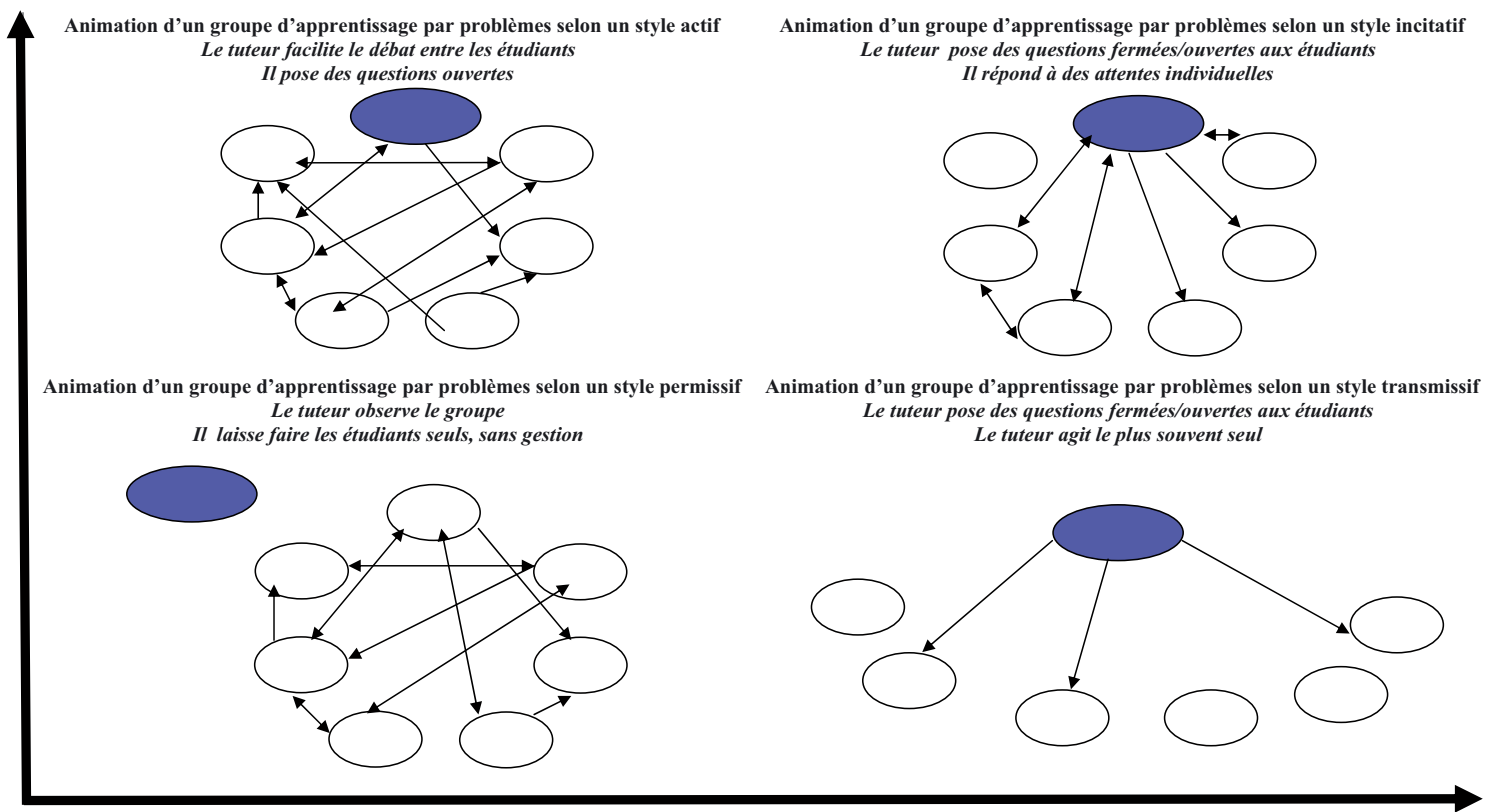

Fig. 2. Styles d'animation dans un dispositif d'apprentissage par problèmes.

d'un tuteur d'APP qui aurait le souci de prendre en compte les principes directeurs, «invariants », de la pédagogie active et qui, pour ce faire, chercherait à développer majoritairement - et judicieusement des pratiques de tutorat correspondant au style actif, en accord avec les orientations pédagogiques actuelles de l'APP.

Le tableau II rappelle les trois grandes phases organisationnelles successives d'un travail dans le cadre d'un dispositif d'APP. Le tableau III préconise une approche pragmatique de la tripartition des rôles lors de chacune des phases dans le cadre d'une animation de style actif au cours d'un dispositif d'APP, en insistant sur le degré de directivité préconisé.

L'exercice proposé est d'identifier qui joue effectivement chacun de ces trois rôles dans l'animation d'un tutorial d'APP. Une observation extérieure systématique et/ou une analyse structurée personnelle de ses propres interventions permet d'engager un processus réflexif. Il est important d'identifier la récurrence de certaines de ses attitudes individuelles tout en prenant conscience de l'impact favorable ou défavorable qu'elles ont pu avoir sur le travail de groupe. L'autoscopie et l'appel à la vidéo sont très porteurs et très formateurs dans ce type de travail. Généralement utilisé dans la formation des psychosociologues, une telle approche pourrait être également proposée aux tuteurs d'APP qui désirent envisager leur animation sous un autre regard.

Le tableau IV propose un support pratique permettant d'identifier le style actif d'animation en APP; il trace les zones de croisement existant entre les concepts issus respectivement des perspectives psychosociologiques et pédagogiques rappelées antérieurement. Il précise les éléments discriminants et évocateurs de ce style d'animation dans le cadre de l'APP, ainsi qu'une liste non exhaustive de pratiques d'animation correspondant au style actif. À partir de cet outil, le tuteur pourra reconnaître le niveau de proximité existant entre son propre style d'animation et celui spécifié dans le tableau IV. Il lui appartient de l'ajuster selon ses choix personnels et contextuels. Ce tableau ne suggère pas un enfermement. Bien au contraire, lorsque les bases d'une méthode sont acquises, on peut s'en éloigner plus facilement et atteindre une plus grande liberté de gestion, tout en respectant le cadre commun à l'ensemble des partenaires de travail. Deleuze ${ }^{[36]}$ 
Tableau I. Tripartition des rôles d'animation au sein des groupes restreints selon une perspective psychosociologique.

\begin{tabular}{|l|l|}
\hline $\begin{array}{l}\text { Le rôle de } \\
\text { production }\end{array}$ & $\begin{array}{l}\text { Il correspond au fait que l'on doit trouver une ou des solutions pertinentes } \\
\text { et valides ou des éléments de solution. À la production s'attache l'exécu- } \\
\text { tion et la validation de la tâche réalisée et des connaissances acquises (en } \\
\text { proposant et illustrant son expertise, en validant des schémas, en avalisant } \\
\text { des solutions en tant qu'expert du sujet évoqué,...). }\end{array}$ \\
\hline $\begin{array}{l}\text { Le rôle de } \\
\text { facilitation }\end{array}$ & $\begin{array}{l}\text { Il correspond à tout ce qui doit être mis en ouvre pour permettre au rôle de } \\
\text { production d'être réalisé. La facilitation aide à la mise en commun des res- } \\
\text { sources intellectuelles des membres du groupe et à la recherche de stratégies } \\
\text { efficaces pour résoudre le problème posé (en proposant des orientations, en } \\
\text { suggérant un plan de travail, en suscitant l'exploration de voies nouvelles, } \\
\text { en évaluant le chemin parcouru par rapport au but final, en aidant à définir } \\
\text { un problème, en faisant expliquer le sens d'une question, ...). . }\end{array}$ \\
\hline $\begin{array}{l}\text { Le rôle de } \\
\text { régulation }\end{array}$ & $\begin{array}{l}\text { Il recouvre explicitement toutes les interventions visant une meilleure faci- } \\
\text { litation et une meilleure production. La régulation consiste plus précisément } \\
\text { à maintenir un climat serein, à apaiser les tensions potentielles et à veiller } \\
\text { à la participation de chacun (en stimulant l'entrain et le dynamisme, en } \\
\text { identifiant l'origine d'un malaise, en encourageant et en témoignant de la } \\
\text { compréhension ... tout en faisant émerger les oppositions et en soulignant } \\
\text { les conflits socio-cognitifs. }\end{array}$ \\
\hline
\end{tabular}

Tableau II. Phases de travail dans un dispositif d'apprentissage par problèmes.

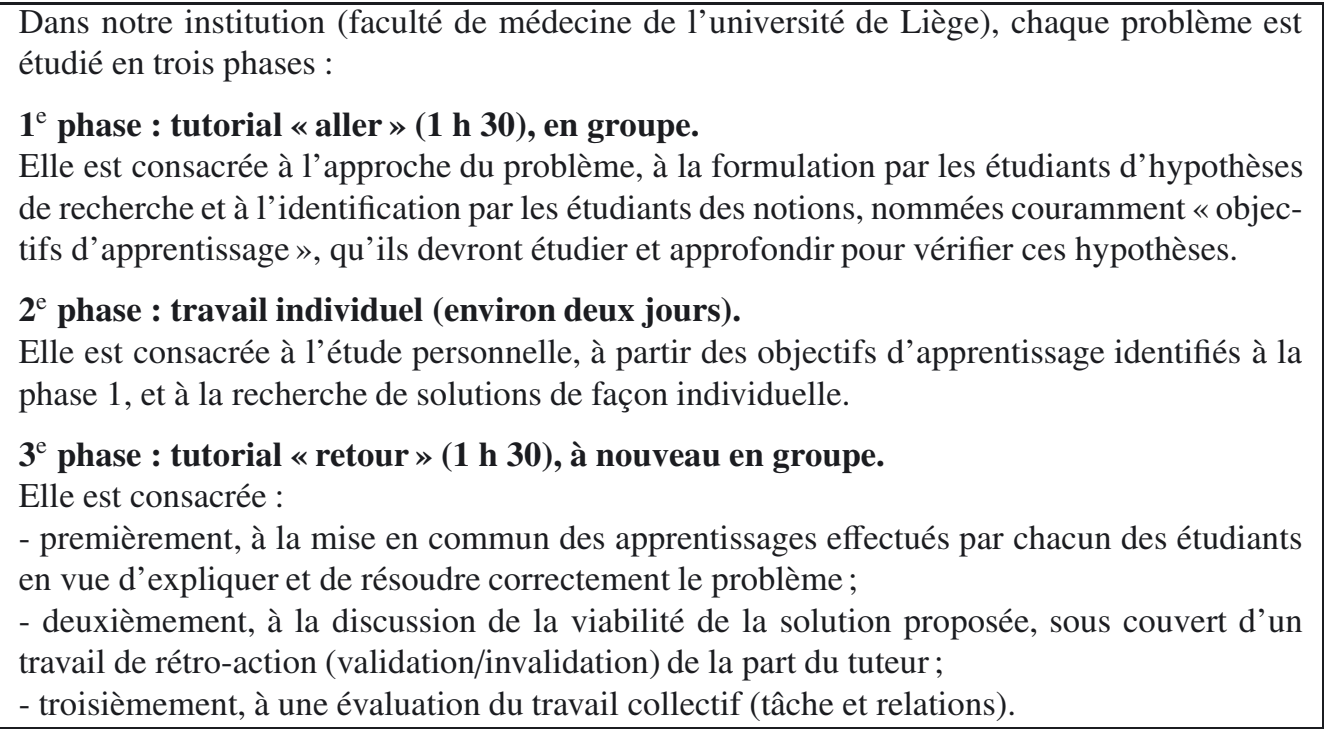

exprime précisément cette intention : «Loin d'être la délimitation de la surface picturale, le cadre est presque le contraire, c'est la mise en relation immédiate avec le dehors ».

Dans une telle perspective, si une pratique du tutorat de style actif est institutionnalisée dans le cadre d'un dispositif d'APP et s'il est proposé aux enseignants de l'appréhender, nous suggérons aux tuteurs de prendre conscience des enjeux qui sont en cause. Dès lors, les principes issus de la perspective psychosociologique pour l'exercice de la fonction d'animation de groupe et des rôles à tenir lors d'un tutorial d'APP nous paraissent fortement utiles. Le processus d'implantation d'un tel changement 
Tableau III. Tripartition des rôles transposée à l'animation d'un groupe selon un style actif dans le cadre d'un dispositif d'apprentissage par problèmes.

\begin{tabular}{|c|c|}
\hline $\begin{array}{l}\text { Le rôle de } \\
\text { production }\end{array}$ & $\begin{array}{l}\text { Les capacités de validation et de production concernant la matière (synthèse/schéma) s'ap- } \\
\text { parentent au rôle de production : } \\
\text { • gérer la production du groupe et contribuer à sa validation; } \\
\text { • faire preuve d'expertise dans la matière; } \\
\text { • être garant des contenus d'apprentissage dans sa discipline personnelle; } \\
\text { - être le garant de l'orientation vers les personnes ressources pour d'autres disciplines; } \\
\text { •... } \\
\text { Lors du tutorial «aller », le tuteur est non directif dans les contenus d'apprentissage (initia- } \\
\text { lement, aucun apport de contenu spécifique). } \\
\text { Lors du tutorial « retour », à l'issue de la mise en commun des travaux individuels, le tu- } \\
\text { teur devient directif car il doit valider/invalider les productions du groupe d'étudiants (voire } \\
\text { diriger vers des experts de la discipline). }\end{array}$ \\
\hline $\begin{array}{l}\text { Le rôle de } \\
\text { facilitation }\end{array}$ & $\begin{array}{l}\text { Les capacités de gestion et de recadrage concernant les stratégies cognitives des étudiants } \\
\text { dans le cadre du processus de résolution de problèmes s'apparentent au rôle de facilitation : } \\
\text { - adopter un comportement directif quant à la forme (organisation et clarification); } \\
\text { - maintenir un comportement non directif quant au fond (matière et contenu); } \\
\text { - organiser les stratégies du développement des connaissances; } \\
\text { - reformuler les idées; } \\
\text { - clarifier les propositions pour faciliter la résolution du problème; } \\
\text { - faire des synthèses; } \\
\text { - définir les limites; } \\
\text { - ouvrir le cadre; } \\
\text { •... } \\
\text { Lors des tutoriaux «aller » et « retour », le tuteur est semi-directif dans la stratégie de réso- } \\
\text { lution de problème. }\end{array}$ \\
\hline $\begin{array}{l}\text { Le rôle de } \\
\text { régulation }\end{array}$ & $\begin{array}{l}\text { Les capacités liées au développement des attitudes sociales favorisant l'interaction et valo- } \\
\text { risant le conflit socio-cognitif entre les étudiants s'apparentent au rôle de régulation : } \\
\text { - adopter un comportement directif favorisant les propositions divergentes/les } \\
\text { désaccords ; conflit socio-cognitif; } \\
\text { - maintenir un comportement non directif quant au fond (matière et contenu); } \\
\text { - contribuer à créer et à maintenir des relations propices à la collaboration de tous en } \\
\text { vue de la résolution de la tâche commune; } \\
\text { - contribuer au meilleur climat socio-affectif dans le groupe (stimule/apaise); } \\
\text { - faire aboutir un conflit latent; } \\
\text { - valoriser des interventions positives; } \\
\text { - réguler la prise de parole; } \\
\text {-... } \\
\text { Lors des tutoriaux «aller» et « retour», le tuteur est semi-directif vis à vis de la communi- } \\
\text { cation collective des étudiants. }\end{array}$ \\
\hline
\end{tabular}




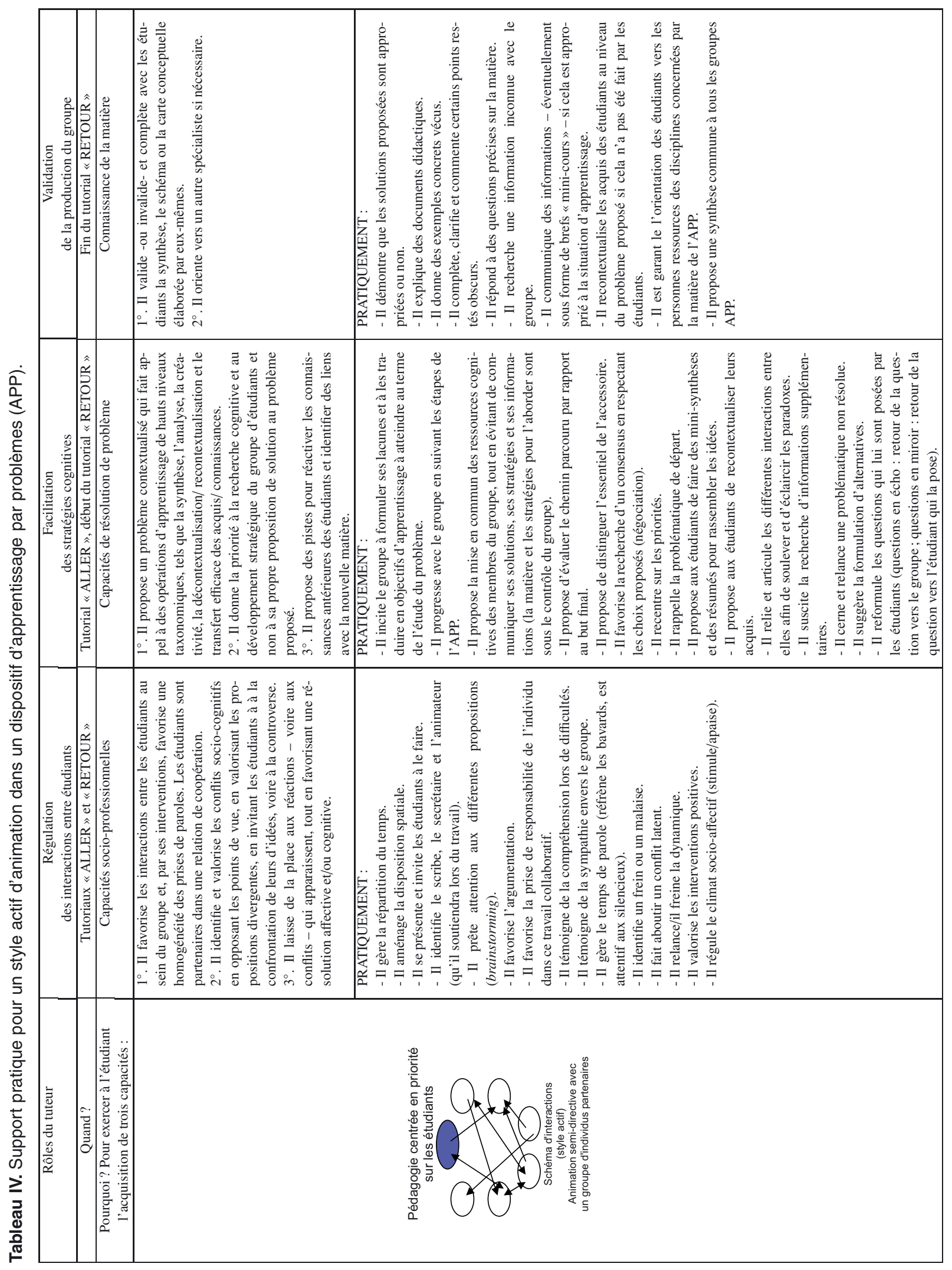


nécessite d'être solidairement partagé par tous les partenaires de travail. C'est à ce prix que les enseignants et les étudiants percevront la cohérence des principes pédagogiques, des décisions institutionnelles et organisationnelles adoptées et des pratiques qu'ils inspirent.

Par ailleurs, le principe d'un recours privilégié au style actif n'exclut pas la sollicitation d'autres styles d'animation pédagogique car il serait illusoire, sinon dogmatique, de se référer à ce seul type de fonctionnement. Ainsi, l'APP de style actif est plus adapté aux petits groupes mais l'EPP de style transmissif se prête mieux aux grands groupes; le style permissif, tel que nous l'avons déjà précisé, s'apparente à une observation extérieure au groupe à des fins d'analyse de la communication ambiante. Un territoire universitaire peut se nourrir de plusieurs pédagogies complémentaires, à la condition qu'une cohérence globale soit préservée et que des signaux paradoxaux ne soient pas simultanément adressés aux étudiants. L'essentiel est de bien identifier les finalités de la formation, les moyens les plus appropriés pour les atteindre, l'évaluation proposée et la faisabilité du projet au sein d'un contexte culturel et institutionnel précis.

\section{Conclusions}

Les méthodes de pédagogie active - dont l'APP constitue un format emblématique - sont fondées prioritairement sur une dynamique plastique et structurée visant à créer les conditions d'apprentissages en profondeur, signifiants et transférables et non sur une mécanique automatique obligée. Leur pratique, qui constitue une rupture par rapport aux pratiques d'enseignement traditionnelles, requiert que les enseignants concernés disposent de ressources adéquates et qu'ils puissent bénéficier d'une formation, d'une supervision et d'un accompagnement adéquats. L'important n'est pas d'utiliser les approches se réclamant de la pédagogie active par principe dogmatique et de manière systématique mais de les utiliser à bon escient pour favoriser l'apprentissage.
Grâce à une prise de conscience des fondements de la pédagogie active, les institutions pourront plus aisément formuler explicitement leurs choix pédagogiques et évoquer des pistes de développement correspondant le mieux à leur environnement et aux contraintes auxquelles elles sont confrontées. Il est important d'insister sur la question du sens des actions pédagogiques. Selon le contexte de travail, les schémas de fonctionnement initiaux peuvent être réinitialisés et remodelés par des stratégies créatives fonctionnelles porteuses d'un sens pédagogique pour l'institution.

Ainsi, on peut admettre que les principes technico-organisationnels usuellement recommandés dans les dispositifs d'APP (organisation des tutoriaux selon des étapes obligatoires, distribution obligatoire de différents rôles aux étudiants, respect d'un temps délimité par phase, production par les étudiants d'un document de synthèse de leurs apprentissages, exclusion du recours aux interventions magistrales - mini-cours - de la part des tuteurs, exclusion de la fourniture systématique de ressources documentaires univoques - notes de cours, syllabus, polycopiés, etc.) n'ont pas vocation à être appliqués de façon rigide et que les tuteurs peuvent développer des pratiques caractérisées par une certaine flexibilité. De telles pratiques doivent cependant être «conscientisées » et mises en œuvre de façon judicieuse et avec rigueur; à cet égard, il est essentiel que les tuteurs aient clairement conscience des principes pédagogiques ou d'animation au nom desquels ils s'autorisent une telle flexibilité.

À l'instar de Mérieu ${ }^{[38]}$, nous estimons «qu'il faut créer, ensemble, des situations pédagogiques qui permettent tout autant la transmission de savoirs structurants que l'émergence de sujets libres et solidaires ». Et, puisqu'il y a maintenant plus que jamais trop de choses à apprendre, de Brabandere ${ }^{[39]}$ conseille «de réinventer une manière d'appréhender les choses pour se préparer à ne pas savoir ce qui va se passer ». Les interventions du tuteur de style actif devraient permettre d'y contribuer de façon significative. 


\section{Contributions}

Viviane Vierset a proposé une première version de l'article en développant notamment les cadres conceptuels pédagogique et psychosociologique. Denis Bédard a enrichi cette première version en introduisant des références pédagogiques complémentaires et a participé à la révision du manuscrit à partir des commentaires du comité de rédaction et des experts. Jean-Michel Foidart a contribué au manuscrit par ses conseils pragmatiques et par son soutien concernant ce projet d'article.

\section{Références}

1. Barrows HS, Tamblyn RM. Problem-Based Learning : An approach to medical education. New York : Springer, 1980.

2. Charlin B, Mann K, Hansen P. The many faces of problembased learning: a framework for understanding and camparison. Med Teach 1998;20:323-30

3. De Theux MN, Jacqmot $\mathrm{Ch}$,Wouters $\mathrm{P}$. Se former à son métier d'étudiant dans un contexte d'apprentissage actif en groupe. Colloque AIPU, 2002. Louvain la Neuve. 2002 [On-line] Disponible sur : http://www. uclouvain. be/202582.html

4. Morin E. La Méthode. La connaissance de la connaissance. Tome 3. Paris : Seuil, 1986.

5. Dewey J. Comment nous pensons. Paris : Flammarion1924.

6. Barr RB, Tagg J. From teaching to Learning : A new paradigm for undergraduate education. In : KA. Feldman \& MB Paulsen : Teaching and Learning in the College Classroom. Boston (MA) : Pearson Custom Publishing, 1998.

7. Anzieu D, Martin J-Y. La dynamique des groupes restreints. Paris : PUF,1968 (4e ed.1979).

8. Vierset V. Sensibilisation à une autre pédagogie. Marrakech : Presse de l'Université UCAM, 2005.

9. Bandura A. Social learning theory. Englewood Cliffs (NJ): Prentice-Hall, 1977.

10. Piaget J. Psychologie et épistémologie. Paris : Denoël, 1967.

11. Vygotsky L. Pensées et langage. Paris : La Dispute, 1934/1997.

12. Bachelard G. La formation de l'esprit scientifique. Paris : Librairie Philosophique Vrin, 1970.
13. Ausubel D. The Psychology of Meaningful Verbal Learning. New York: Grune \& Stratton,1963.

14. Leung KK, Lue BH, Lee MB. Development of a teaching style inventory for tutor evaluation in problem-based learning. Med Educ 2003;7:410-6.

15. Blake R, Mouton J. The Managerial Grid: The Key to Leadership Excellence. Houston: Gulf Publishing Co, 1964 .

16. Therer J, Willemart Cl. Styles et stratégies d'animation pédagogique. Notes de cours de pédagogie pour adultes. Liège : Faculté de psychologie, 1990.

17. Spronken-Smith R, Harland T. Learning to teach with problem-based learning. Active Learning in Higher Education 2009;10:138-53.

18. Bowman D, Hughes P. Emotional responses of tutors and students in problem-based learning : lessons for staff development. Med Educ 2005;39:145-53.

19. Albanese M. Treading tactfully on tutor turf: does PBL tutor content expertise make a difference? Med Educ 2004;38:918-920.

20. Steinert Y. Student perceptions of effective small group teaching. Med Educ 2004;38:286-93.

21. Mete S, Sari HY. Nursing students'expectations from tutors in PBL and effects of tutors' behaviour on nursing students. Nurse Educ Today 2008;28:434-42.

22. Caplow JH, Donaldson JF, Kardash CA, Hosokawa M. Learning in a Problem-Based Medical Curriculum: Students' Conceptions. Med Educ 1997;31:440-7

23. Silver M,Wilkerson L. Effects of tutors with subject-matter expertise on the problem-based tutorial process. Acad Med 1991;66:298-300

24. Gilkinson A. Techniques used by "expert" and "nonexpert" tutors to facilitate problem-based learning tutorials in an undergraduate medical curriculum. Med Educ 2003;37:6-14

25. Cooper MH. An exploration of tutors' experiences of facilitating problem-based learning. Part 2 - Implications for the facilitation of problem-based learning. Nurse Educ Today 2003;23:65-75

26. McLean M. What can we learn from facilitator and student perceptions of facilitation skills and roles in the first year of a problem-based learning curriculum? BMC Med Educ 2003;3:9

27. De Grave WS, Dolmans DH, Van Der Vleuten CPM. Profiles of effective tutors in problem-based learning: scaffolding student learning. Med Educ 1999;33:901-6.

28. Lebrun M. Théories pédagogiques pour enseigner et apprendre ? Bruxelles : De Boeck, 2007. 
29. Bédard D, Frenay, M. Turgeon J, Paquay L. Les fondements de dispositifs pédagogiques visant à favoriser le transfert de connaissances : les perspectives de "l'apprentissage et de l'enseignement contextualisés authentiques". Res Academica 2000 ;18:21-47

30. Frenay M, Bédard D. Des dispositifs de formation universitaire s'inscrivant dans la perspective d'un apprentissage et d'un enseignement contextualisés pour favoriser la construction de connaissances et leur transfert. In : A Presseau et M Frenay (dir.) : Le transfert des apprentissages. Québec : Les Presses de l'Université Laval, 2004.

31. Papinczak T, Tunny T, Young L. Conducting the symphony : a qualitative study of the facilitation in problembased learning tutorials. Med Educ 2009;43:377-83

32. Frenay M, Bédard D. Le transfert des apprentissages. In : Bourgeois et Chapelle G (dir.). Apprendre et faire apprendre. Paris : PUF, 2006.

33. Beser A, Mete S, Yildirim HS. How should be a tutor in a problem-based learning ? Journal of Cumhuriyet University School of Nursing 2004;8:32-8

34. Dolmans DH, Wolfhagen IH, Schmidt HG, Van Der Vleuten CPM. (2002). A rating scale for tutor evaluation in a problem-based curriculum: validity and reliability. Med Educ 2002;28:550-8

35. Palmade G. La fonction et les rôles d'animateur de goupes restreints. In : R. Delhez. Cours de dynamique des groupes restreints. Liège : Faculté de psychologie, 1990.

36. Saint Arnaud Y. Le changement assisté. Montréal-Paris : Gaétan Morin, 1999.

37. Deleuze G. L'île déserte et autres textes. Paris : Ed. de Minuit 2002.

38. Meirieu Ph. Pédagogie : Le devoir de résister. Paris, ESF, 2008

39. De Brabandère L. Le sens des idées. Pourquoi la créativité ? Paris : Dunod, 2004.

Correspondance et offprints : Viviane VIERSET, Faculté de médecine Université de Liège, Service Gynécologie-obstétrique (Professeur J-M Foidart), Hôpital de la Citadelle boulevard du $12^{\mathrm{e}}$ de Ligne, 1 (J36) 4000 Liège, Belgique Mailto : viviane.vierset@chrcitadelle.be 